\title{
Prevalence of Adverse Intraoperative Events during Obesity Surgery and Their Sequelae
}

\author{
Alexander J Greenstein, MD, MPH, Abdus S Wahed, PhD, Abidemi Adeniji, MS, Anita P \\ Courcoulas, MD, MPH, FACS, Greg Dakin, MD, FACS, David R Flum, MD, MPH, FACS, \\ Vincent Harrison, MD, James E Mitchell, MD, Robert O'Rourke, MD, FACS, Alfons Pomp, \\ MD, FACS, John Pender, MD, FACS, Ramesh Ramanathan, MD, and Bruce M Wolfe, MD, \\ FACS \\ From the Department of General Surgery, Mount Sinai School of Medicine (Greenstein), \\ Department of Surgery, Weill Cornell Medical College (Dakin, Pomp), New York, NY, Department \\ of Biostatistics, University of Pittsburgh (Wahed, Adeniji), Division of Minimally Invasive Bariatric \\ and General Surgery, Department of Surgery, University of Pittsburgh Medical Center \\ (Courcoulas, Ramanathan), Pittsburgh, PA, Department of General Surgery, University of \\ Washington, Seattle, WA (Flum), Department of General Surgery, Oregon Health and Science \\ University, Portland, OR (Harrison, O'Rourke, Wolfe), Neuropsychiatric Research Institute, \\ University of North Dakota, Fargo, ND (Mitchell), and Department of Surgery, East Carolina \\ University, Greenville, NC (Pender)
}

\section{Abstract}

BACKGROUND—Adverse intraoperative events (AIEs) during surgery are a well-known entity. A better understanding of the incidence of AIEs and their relationship with outcomes is helpful for surgeon preparation and preoperative patient counseling. The goals of this study are to describe the incidence of AIEs during bariatric surgery and examine their impact on major adverse complications.

STUDY DESIGN-The study included 5,882 subjects who had bariatric surgery in the Longitudinal Assessment of Bariatric Surgery study between March 2005 and April 2009.

Prospectively collected AIEs included organ injuries, anesthesia-related events, anastomotic revisions, and equipment failure. The relationship between AIEs and a composite end point of 30day major adverse complications (ie, death, venous thromboembolism, percutaneous, endoscopic, or operative reintervention and failure to be discharged from the hospital within 30 days from surgery) was evaluated using a multivariable relative risk model adjusting for factors known to influence their risk.

RESULTS-There were 1,608 laparoscopic adjusted gastric banding, 3,770 laparoscopic Rouxen-Y gastric bypass operations, and 504 open Roux-en-Y gastric bypass operations. Adverse intraoperative events occurred in 5\% of the overall sample and were most frequent during open

\footnotetext{
(C) 2012 by the American College of Surgeons

Correspondence address: Alexander J Greenstein, MD, MPH, Department of General Surgery, The Mount Sinai Medical Center, $5 \mathrm{E} 98^{\text {th }}$ St, Box 1259, $15^{\text {th }}$ Fl, New York, NY 10029. Alexander.Greenstein@ mountsinai.org.

Disclosure Information: Dr Courcoulas received a research grant from Allergan; Dr Dakin is a paid consultant for Covidien; Dr Flum received a research grant from Covidien; Dr Mitchell received a research grant from Lilly; Dr Pender is a paid consultant and received a research grant from Covidien; and Dr Wolfe is a paid consultant for Allergan. All other authors have nothing to disclose.

Author Contributions Study conception and design: Greenstein, Wahed, Adeniji, Wolfe

Acquisition of data: Wahed, Adeniji, Courcoulas, Dakin, Flum, Mitchell, O'Rourke, Pomp, Pender, Ramanathan, Wolfe

Analysis and interpretation of data: Greenstein, Wahed, Adeniji, Courcoulas, Dakin, O'Rourke, Pender, Ramanathan, Wolfe

Drafting of manuscript: Greenstein, Wahed, Adeniji, Harrison, Wolfe

Critical revision: Courcoulas, Dakin, Flum, Harrison, Mitchell, O'Rourke, Pomp, Pender, Ramanathan, Wolfe
} 
Roux-en-Y gastric bypass (7.3\%), followed by laparoscopic Roux-en-Y gastric bypass (5.5\%) and laparoscopic adjusted gastric banding (3\%). The rate of composite end point was $8.8 \%$ in the AIE group compared with $3.9 \%$ among those without an AIE ( $p<0.001$ ). Multivariable analysis revealed that patients with an AIE were at $90 \%$ greater risk of composite complication than those without an event (relative risk $=1.90 ; 95 \%$ CI, 1.26-2.88; $\mathrm{p}=0.002$ ), independent of the type of procedure (open or laparoscopic).

CONCLUSIONS-Incidence of an AIE is not infrequent during bariatric surgery and is associated with much higher risk of major complication. Additional study is needed to assess the association between specific AIEs and short-term complications.

In the past few years, research on the benefits of bariatric surgery for several medical comorbidities as well as for overall mortality have moved beyond single-institution series to include a randomized controlled trial, a large matched cohort study, and a meta analysis. ${ }^{1-4}$ With multiple reports about its positive effects on comorbid conditions and overall mortality in the obese population, bariatric surgery has been used with increasing frequency as a primary intervention for weight loss. The combination of shifting perceptions and improved access and coverage has resulted in a markedly increased volume in the number of bariatric procedures being performed. With a greater need for information on the safety and efficacy of bariatric surgery, the Longitudinal Assessment of Bariatric Surgery (LABS) consortium was established by the National Institute of Diabetes, Digestive and Kidney Diseases in the National Institutes of Health. ${ }^{5}$

The LABS was designed as a prospective, multicenter observational study to evaluate short and long-term safety and efficacy outcomes in patients undergoing bariatric surgical procedures at 10 clinical sites in the United States (2005-2007). An initial report focused on the incidence of 30-day adverse outcomes and the patient-specific factors associated with them. Overall risks of death and other adverse outcomes after bariatric surgery were found to be low but correlated with patient factors such as a history of deep vein thrombosis (DVT) or pulmonary embolus (PE), a diagnosis of obstructive sleep apnea, and impaired functional status. ${ }^{6}$

Although knowledge about the major postoperative complications of bariatric surgery has improved greatly, much less is known about adverse intraoperative events (AIEs) during bariatric surgery. These include occurrences such as such as organ injuries, intraoperative bleeding, anesthesia-related events, anastomotic revisions (Rouxen-Y gastric bypass only), and equipment failure. These AIEs are believed to be more common than other adverse events, but little is known about their incidence, associations, and sequelae.

The objectives of this study were to use well-designed, prospective data from the LABS study to describe the incidence of AIEs during bariatric surgery and to examine the impact of AIEs on an established composite end point (CE) of 30-day major adverse complications. Specifically, we hypothesized that AIEs are associated with higher risks of major adverse complications after adjusting for other factors known to influence their risk.

\section{METHODS}

\section{Data source and selection of study population}

The LABS is a longitudinal observational study designed to assess the risks and benefits of bariatric surgery and is organized into 3 phases: LABS-1, LABS-2, and LABS-3. ${ }^{5}$ LABS- 1 consists of all patients at least 18 years of age who underwent bariatric surgery from March 11, 2005 through December 31, 2007, performed by 1 of 33 LABS-certified surgeons at participating centers. The Institutional Review Board at each institution approved the LABS-1 protocol and consent form. By the end of 2007, a total of 5,648 patients had agreed 
to participate in the study, and 4,776 had undergone primary operations. LABS- 1 consists of a limited dataset of patient and operative characteristics and was crafted to evaluate the short-term safety of bariatric surgery. Primary end points include important adverse outcomes, such as death and/or operative reintervention within 30 days of surgery. The LABS-1 inclusion criteria and data collection have been described in detail previously. ${ }^{5}$

The primary goal of LABS-2 is to evaluate the longer-term safety and efficacy of bariatric surgery and better study patient characteristics as they relate to short and intermediate-term outcomes. Key groups of nonsafety outcomes for LABS-2 include (but are not limited to) weight loss, changes in body composition, functional impairment, psychosocial function (including quality of life), and cardiovascular, metabolic, pulmonary, renal, musculoskeletal, urogynecologic, reproductive, and gastrointestinal outcomes. The sample size for LABS-2 is approximately 2,400 patients and data collection consists of an array of demographic, anthropometric, clinical, behavioral, surgical, and postoperative care variables. Data are collected before surgery, during surgery, and postoperatively at multiple intervals including 30 days, 6 months, 1 year, and annually thereafter. The LABS-2 protocol and consent form were approved by the Institutional Review Board at each institution and details of the LABS-2 evaluations have been reported previously. Recruitment of patients occurred between February 2006 and February 2009. Participants who proceeded to surgery were eligible to be included in these initial analyses. ${ }^{5}$ LABS- 3 study involves additional subsets, determined in composition and size by the hypotheses underlying the mechanisms to be studied, of patients from the LABS-2 cohort. One LABS-3 study measures the psychosocial and behavioral aspects of obesity, such as quality of life, and a second LABS-3 study examines the mechanisms underlying diabetes resolution. ${ }^{5}$

This study included 5,882 patients undergoing one of the following types of primary bariatric operations in LABS-1 or LABS-2: laparoscopically adjusted gastric banding (LAGB), laparoscopic Roux-en-Y gastric bypass (LRYGB), or open Roux-en-Y gastric bypass (ORYGB). Of the 5,882 patients, 4,609 patients were recruited as part of LABS-1 and 2,348 were part of LABS-2. Note that of the 4,609 patients, 1,057 patients also participated in LABS-2.

\section{Data points and statistical analysis}

Participating surgeons completed a questionnaire after the surgery performed and among multiple variables there were questions about the occurrences of AIEs. Adverse intraoperative events data were collected somewhat differently for LABS-1 and LABS-2. Both the common injury data elements for all 5,882 patients and other study-specific data elements unique to LABS-1 and LABS-2 patients will be described. These include organ (ie, liver, spleen, blood vessel, bowel, and diaphragm) injuries, bleeding $\geq 2 \mathrm{U}$, anesthesiarelated events (multiple intubation attempts, sustained dysrhythmia, sustained hypoxia, sustained hypotension, and sustained hypercarbia), anastomotic revisions (gastrojejunostomy, jejunojejunostomy, unspecified), and instrument/equipment failure (such as staple misfire). Specifically, an organ injury was documented if there was unanticipated repair of a damaged internal organ by use of sutures or other material, and a blood vessel injury was documented if there was unexpected injury to a large artery or vein with specific anatomic designation (eg, renal artery). In addition to postoperative mortality, data were collected on the CE of 30-day major adverse complications (eg, death, venous thromboembolism, percutaneous, endoscopic, or operative reintervention and failure to be discharged from the hospital within 30 days from surgery) as reported previously. ${ }^{6}$

The patient characteristics have been described using summary statistics, such as frequencies and percentages. Rates of AIE across types of operations (LAGB, LRYGB, and ORYGB) were compared using Pearson chi-square test. Unadjusted association between 
incidence of AIE and major complications, as well as the CE, were examined using Fisher's exact test. The relationship between AIEs and CE was evaluated using a multivariable relative risk model adjusting for DVT/PE, obstructive sleep apnea, body mass index (BMI; calculated as $\mathrm{kg} / \mathrm{m}^{2}$ ), procedure type, inability to walk 200 feet, surgeon volume, and clustering due to surgeon and site. For all statistical analyses, Statistical Analysis Systems, version 9.2 (SAS Institute), was used. A p $<0.05$ was used to determine statistical significance.

\section{RESULTS}

A total of 5,882 patients were included in the study. There were 1,608 LAGB (27\%), 3,770 LRYGB (64\%), and 504 ORYGB (9\%) operations. Median age was 47 years, $76.3 \%$ were female, $89.7 \%$ were Caucasian, and 5.0\% Hispanic, with a median BMI of 44. Complete demographic information by operative type is listed in Table 1. Compared with patients having LRYGB, patients having ORYGB were relatively heavier (median BMI 51.0 vs 46.7) and older (median 47 vs 44 years); included more males (32.3\% vs $18.1 \%$ ), Caucasians (92.2\% vs $87.6 \%$ ), and non-Hispanics (96.4\% vs 93.9\%; all, p < 0.04 ). Two of the 10 sites conducted most of the open procedures. Of the 3,838 cases that started as laparoscopic, only 68 (1.8\%) needed to be converted to open.

Adverse intraoperative events occurred in $294(5 \%)$ of the overall patients. Of these 294 patients, $264(89.8 \%)$ had one, $25(8.5 \%)$ had 2 , and $3(1.0 \%)$ had $<2$ intraoperative events. Data on the specifics of the AIEs were missing for 2 patients. The most common AIEs were organ injuries (1.6\%), followed by anesthesia events $(0.9 \%)$ and equipment failure $(0.8 \%)$. Anastomosis revisions were required in $0.6 \%$ of patients. The most common form of injury was bowel injury $(0.9 \%)$, followed by liver injury $(0.4 \%)$ and spleen injury $(0.2 \%)$. Adverse intraoperative events were most frequent for ORYGB (7.3\%), followed by LRYGB (5.5\%) and LAGB (3\%). Table 2 shows the rate of intraoperative injuries by procedure for the patients included in this analysis and contains the common categories of intraoperative injuries in LABS-1 that were expanded in LABS-2. Specific injuries that are not collected in LABS- 1 but are collected in LABS- 2 are not presented for the overall sample. The major individual events that are reported in Table 2 merge events that are collected the same way or could be mapped between 2 studies for all patients. The difference in the rates of AIE between the LRYGB and ORYGB groups was not statistically significant $(\mathrm{p}=0.13)$, and the combined rate of AIE in RYGB was significantly higher than that in LAGB (5.8\% vs 3\%; p $<0.001$ ). More detailed information on AIEs separated by individual LABS studies (LABS-1 and LABS-2) can be found in Supplementary Tables 1 and 2 (available online only). The incidence of specific AIEs (eg, instrument/equipment failure, bowel injury) was too rare to conduct a valid statistical comparison across different procedures. For the same reason, a comparison of short-term complications across occurrence of specific injuries could not be done.

\section{Univariate associations between adverse intraoperative events and major surgical complications}

The relationship between 30-day major adverse complications and the incidence of an AIE is shown in Table 3. Although incidence of death $(0.3 \%)$ and DVT/PE $(0.4 \%)$ were similar $(\mathrm{p}>0.05)$ across those with or without an AIE, abdominal reoperation $(4.8 \%$ vs $2.4 \%$; $\mathrm{p}=$ $0.01)$, percutaneous drain placement $(1.0 \%$ vs $0.3 \% ; \mathrm{p}=0.02)$, and endoscopic intervention ( $2.4 \%$ vs $1.1 \% ; \mathrm{p}=0.04)$ were more common among those with an AIE. The rate of CE (death; DVT or venous thromboembolism; reintervention with the use of a percutaneous, endoscopic, or operative technique; or failure to be discharged from the hospital within 30 days after surgery) was $8.8 \%$ among those with an AIE compared with $3.9 \%$ among those 
without an AIE ( $\mathrm{p}<0.001)$. Patients with an AIE were $>2 \times$ more likely to have short-term complications than those without an AIE.

Composite end point rates were compared across various types of AIEs, but the incidence of both specific AIEs and CEs were so infrequent that it was not possible to conduct a statistically powered analysis to assess this association. In Figure 1, CE rates and the corresponding 95\% confidence intervals in the 5 major categories of AIEs (ie, bowel injury, other organ injuries, anesthesia events, equipment failure, and anastomosis revision) can be seen. Composite end point rates were highest (19.2\%; 95\% CI, 8.5-29.9\%) for bowel injury followed by $8.1 \%$ (95\% CI, $0-16.9 \%$ ) for anastomosis revision, but the rates were similar for anesthesia events, instrument failure, and organ injury. As the confidence intervals overlap, the difference in CE rates across these 5 major AIE categories is not statistically significant.

\section{Multivariable associations between adverse intraoperative events and major surgical complications}

Table 4 shows that AIEs remained strongly correlated with major adverse events even after controlling for other key prognostic factors (ie, history of DVT/PE, obstructive sleep apnea, BMI, procedure type, inability to walk 200 feet, and surgeon volume) and accounting for clustering due to surgeon and site. The relationship between BMI and CE was quadratic, with rate of CE being higher for subjects with extreme (too low or too high) BMI compared with others. Multivariable analysis revealed that patients with an AIE were at $90 \%$ greater risk of composite complication than those without an event (relative risk $=1.90 ; 95 \% \mathrm{CI}$, $1.26-2.88 ; \mathrm{p}=0.002$ ).

\section{DISCUSSION}

In this longitudinal observational study of 5,882 patients who underwent 1 of 3 bariatric procedures (ie, LAGB, LRYGB, or ORYGB), we found that 1 in 20 patients had an AIE, the most common of which were organ injuries, anesthesia events, and equipment failure. Although AIEs such as these, once resolved, are typically considered to have little or no consequence, we found that patients with an AIE had a nearly 2-fold greater chance of having a major postoperative complication.

With regard to surgical approach, as expected, there was a trend toward a greater incidence of AIEs in the RYGB groups as a whole when compared with the LAGB group, but the rate of AIEs in the LRYGB group was similar to that found in the ORYGB group. When assessing individual AIEs, such as intraoperative leaks or iatrogenic organ injuries, we were only able to detect trends in favor of the laparoscopic approach, but did not find any statistically significant differences.

This is the first study to demonstrate a multivariable relationship between AIEs and major surgical complications of obesity surgery or any other surgical subspecialty. Although there have been isolated case reports of splenic and portal vein injuries during bariatric surgery, 7,8 overall reporting of intraoperative events or injuries and their sequelae is sparse. The vast majority of reports, especially those based on administrative data, focus on complications that have been documented in the immediate postoperative period, not documented during the operation itself. Even in well-designed cohort studies such as the Michigan Bariatric Surgery Collaborative Study, data for the clinical registry is collected via medical record review, ${ }^{9,10}$ which would allow most intraoperative events to go undocumented unless specifically noted in the medical record by the surgical team. Although a few studies have looked into the specific question of the detection and management of intraoperative leaks ${ }^{11-15}$ and revealed good postoperative results after the leaks have been addressed, these 
are retrospective single-institution studies with limited scope. In comparison, LABS was designed to explore all potential intraoperative events in a protocol-defined, systematic, and prospective fashion.

With regard to the association detected between AIEs and postoperative complications, there are several potential explanations. First, both types of outcomes could be correlated because the risks of AIEs and major complications are related to other underlying patient factors. For example, if a case were technically challenging due to unusual anatomy, there might be a greater chance of a spleen injury or anastomotic revision, and a similarly increased requirement for reoperation or drain placement.

Second, both AIEs and major complications might be related to the overall skill and quality of the surgical approach and/or perioperative management. Extremely skilled (proficient) surgeons and surgical teams would be expected to possess superior technical and cognitive skills and resources that decrease the chance of inadvertent injuries or equipment failure as well as lower overall postoperative complication rates. As such, the rate of AIEs might be seen as a surrogate marker of overall quality of surgical care at either the surgeon or hospital level.

Third, there might be occasions where there is a direct causal link, that is, the AIE causes a postoperative complication. In rare cases, an anastomosis requiring revision could ultimately have a postoperative leak, leading to drain placement and/or reoperation. It is most likely that a combination of these different pathways could contribute to the overall relationship between AIEs and postoperative complications. Better understanding of these relationships will require additional investigation.

Although our study has several strengths, most important of which is that it is large, multicenter, and prospective, it is worth commenting on a few limitations. First, it depended on documentation of AIEs and complications in the study questionnaires. Because we evaluated outcomes of bariatric surgery in the real world, it is possible that some AIEs or postoperative complications were not documented. There was no consistent way, for example, to detect intraoperative leaks, as the surgeon was able to use whatever technique he/she preferred. In addition, our results are derived from Centers of Excellence in bariatric surgery and whether our findings would be replicated in the general community is unknown.

\section{CONCLUSIONS}

We found that AIEs are not uncommon during bariatric surgery and are associated with a much higher risk of postoperative complication. Future work is needed to better understand the reason for these associations, as well as to identify potential interventions to reduce their occurrence.

\section{Supplementary Material}

Refer to Web version on PubMed Central for supplementary material.

\section{Acknowledgments}

This clinical study was a cooperative agreement funded by the National Institute of Diabetes and Digestive and Kidney Diseases. Grant numbers: DCC U01 DK066557; Columbia U01-DK66667 (in collaboration with Cornell University Medical Center CTRC, Grant UL1-RR024996); University of Washington U01-DK66568 (in collaboration with CTRC, Grant M01RR-00037); Neuropsychiatric Research Institute U01-DK66471; East Carolina University U01-DK66526; University of Pittsburgh Medical Center U01-DK66585 (in collaboration with CTRC, Grant UL1-RR024153); and Oregon Health and Science University U01-DK66555. 


\section{Appendix}

Longitudinal Assessment of Bariatric Surgery personnel contributing to the study include:

Columbia University Medical Center, New York, NY: Paul D Berk, MD, Marc Bessler, MD, Amna Daud, Harrison Lobdell IV, Jemela Mwelu, Beth Schrope, MD, PhD, Akuezunkpa Ude, MD

Cornell University Medical Center, New York, NY: Michelle Capasso, BA, Ricardo Costa, BS, Greg Dakin, MD, Faith Ebel, RD, MPH, Michel Gagner, MD, Jane Hsieh, BS, Alfons Pomp, MD, Gladys Strain, PhD

Mt Sinai Medical Center, New York, NY: W Barry Inabnet, MD

East Carolina Medical Center, Greenville, NC: Rita Bowden, RN, William Chapman, MD, FACS, Lynis Dohm, PhD, John Pender, MD, Walter Pories, MD, FACS

Neuropsychiatric Research Institute, Fargo, ND: Jennifer Barker, MBA, Michael Howell, MD, Luis Garcia, MD, FACS, MBA, Kathy Lancaster, BA, Erika Lovaas, BS, James E Mitchell, MD, Tim Monson, MD

Oregon Health and Science University: Chelsea Cassady, BS, Clifford Deveney, MD, Katherine Elder, PhD, Andrew Fredette, BA, Stefanie Greene, Jonathan Purnell, MD, Robert O'Rourke, Lynette Rogers, MD, Chad Sorenson, Bruce M Wolfe, MD

Legacy Good Samaritan Hospital, Portland, OR: Emma Patterson, MD, Mark Smith, MD, William Raum, MD, Lisa VanDerWerff, PAC, Jason Kwiatkowski, PAC, Jamie Laut

Sacramento Bariatric Medical Associates, Sacramento, CA: Iselin Austrheim-Smith, CCRP, Laura Machado, MD

University of Pittsburgh Medical Center, Pittsburgh, PA: Chris Costa, BA, Anita P Courcoulas, MD, MPH, FACS, Jessie Eagleton, BS, George Eid, MD, William Gourash, MSN, CRNP, Lewis H Kuller, MD, DrPH, Carol A McCloskey, MD, Ramesh Ramanathan, MD, Rebecca Search, MPH, Eleanor Shirley, MA

University of Washington, Seattle, WA: David E Cummings, MD, E Patchen Dellinger, MD, Hallie Ericson, BA, David R Flum, MD, MPH, Katrina Golub, MPH, CCRC, Brant Oelschlager, MD, Skye Steptoe, MS, CCRC, Tomio Tran, Andrew Wright, MD

Virginia Mason Medical Center, Seattle, WA: Lily Chang, MD, Stephen Geary, RN, Jeffrey Hunter, MD, Anne MacDougall, BA, Ravi Moonka, MD, Olivia A Seibenick, CCRC, Richard Thirlby, MD

Data Coordinating Center, Graduate School of Public Health at the University of Pittsburgh, Pittsburgh, PA: Abi Adenijii, MS, Steven H Belle, PhD, MScHyg, Lily (Jia-Yuh) Chen, MS, Michelle Fouse, BS, Jesse Hsu, MS, Wendy C King, PhD, Kevin Kip, PhD, Kira Leishear, BS, Laurie Iacono, MFA, Debbie Martin, BA, Rocco Mercurio, MBA, Faith Selzer, PhD, Abdus Wahed, PhD

National Institute of Diabetes and Digestive and Kidney Diseases: Mary Evans, PhD, Mary Horlick, MD, Carolyn W Miles, PhD, Myrlene A Staten, MD, Susan Z Yanovski, MD

National Cancer Institute: David E Kleiner, MD, PhD 


\section{Abbreviations and Acronyms}

$\begin{array}{ll}\text { AIE } & \text { adverse intraoperative events BMI } \\ \text { BMI } & \text { body mass index CE } \\ \text { CE } & \text { composite end point } \\ \text { DVT } & \text { deep vein thrombosis } \\ \text { LABS } & \text { Longitudinal Assessment of Bariatric Surgery } \\ \text { LAGB } & \text { laparoscopic adjusted gastric banding } \\ \text { LRYGB } & \text { laparoscopic Roux-en-Y gastric bypass } \\ \text { ORYGB } & \text { open Roux-en-Y gastric bypass } \\ \text { PE } & \text { pulmonary embolus }\end{array}$

\section{REFERENCES}

1. Sjostrom L, Narbro K, Sjostrom CD, et al. Effects of bariatric surgery on mortality in Swedish obese subjects. N Engl J Med. 2007; 357:741-752. [PubMed: 17715408]

2. Adams TD, Gress RE, Smith SC, et al. Long-term mortality after gastric bypass surgery. N Engl J Med. 2007; 357:753-761. [PubMed: 17715409]

3. O'Brien PE, Dixon JB, Laurie C, et al. Treatment of mild to moderate obesity with laparoscopic adjustable gastric banding or an intensive medical program: a randomized trial. Ann Intern Med. 2006; 144:625-633. [PubMed: 16670131]

4. Buchwald H, Avidor Y, Braunwald E, et al. Bariatric surgery: a systematic review and metaanalysis. JAMA. 2004; 292:1724-1737. [PubMed: 15479938]

5. Belle SH, Berk PD, Courcoulas AP, et al. Safety and efficacy of bariatric surgery: Longitudinal Assessment of Bariatric Surgery. Surg Obes Relat Dis. 2007; 3:116-126. [PubMed: 17386392]

6. Longitudinal Assessment of Bariatric Surgery (LABS) Consortium. Flum DR, Belle SH, et al. Perioperative safety in the longitudinal assessment of bariatric surgery. N Engl J Med. 2009; 361:445-454. [PubMed: 19641201]

7. Huerta S, Li Z, Livingston EH. Outcome of portal injuries following bariatric operations. Obes Surg. 2006; 16:105-109. [PubMed: 16417768]

8. Peters TG, Steinmetz SR, Cowan GS Jr. Splenic injury and repair during bariatric surgical procedures. South Med J. 1990; 83:166-169. [PubMed: 2406932]

9. Birkmeyer NJ, Dimick JB, Share D, et al. Hospital complication rates with bariatric surgery in Michigan. JAMA. 2010; 304:435-442. [PubMed: 20664044]

10. Birkmeyer NJ, Share D, Campbell DA Jr, et al. Partnering with payers to improve surgical quality: the Michigan plan. Surgery. 2005; 138:815-820. [PubMed: 16291379]

11. Madan AK, Martinez JM, Lo Menzo E, et al. Omental reinforcement for intraoperative leak repairs during laparoscopic Roux-en-Y gastric bypass. Am Surg. 2009; 75:839-842. [PubMed: 19774958]

12. Cingi A, Yavuz Y. Intraoperative endoscopic assessment of the pouch and anastomosis during laparoscopic Roux-en-Y gastric bypass. Obes Surg. 2011; 21:1530-1534. [PubMed: 21229330]

13. Kligman MD. Intraoperative endoscopic pneumatic testing for gastrojejunal anastomotic integrity during laparoscopic Roux-en-Y gastric bypass. Surg Endosc. 2007; 21:1403-1405. [PubMed: 17332963]

14. Sekhar N, Torquati A, Lutfi R, et al. Endoscopic evaluation of the gastrojejunostomy in laparoscopic gastric bypass. A series of 340 patients without postoperative leak. Surg Endosc. 2006; 20:199-201. [PubMed: 16333555]

15. Shin RB. Intraoperative endoscopic test resulting in no postoperative leaks from the gastric pouch and gastrojejunal anastomosis in 366 laparoscopic Roux-en-Y gastric bypasses. Obes Surg. 2004; 14:1067-1069. [PubMed: 15479595] 


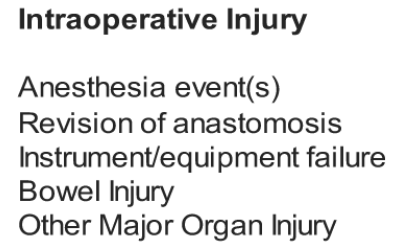

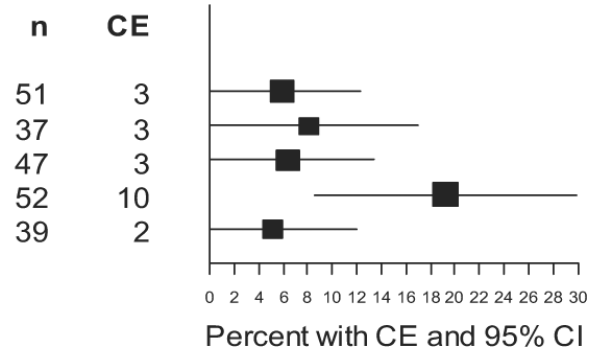

Figure 1.

Composite end point (CE) rates and $95 \%$ confidence intervals by major intraoperative events. 


\section{Table 1}

Demographics by Surgical Approach for Longitudinal Assessment of Bariatric Surgery 1 or Longitudinal Assessment of Bariatric Surgery 2 Participants

\begin{tabular}{|c|c|c|c|c|}
\hline Characteristic & Lap band $(n=1,608)$ & Lap GBP $(n=3,770)$ & Open GBP $(n=504)$ & p Value \\
\hline Age, y, median (Q1, Q3) & $47.0(37.0,56.0)$ & $44.0(36.0,53.0)$ & $47.0(39.0,55.0)$ & $<0.0001$ \\
\hline Age group, y, n (\%) & & & & $<0.0001$ \\
\hline$<30$ & $164(10.2)$ & $382(10.1)$ & $40(7.9)$ & \\
\hline $30-39$ & $335(20.8)$ & $1,010(26.8)$ & $100(19.8)$ & \\
\hline $40-49$ & $435(27.1)$ & $1,098(29.1)$ & $153(30.4)$ & \\
\hline $50-59$ & $407(25.3)$ & $950(25.2)$ & $153(30.4)$ & \\
\hline $60+$ & $267(16.6)$ & $330(8.8)$ & $58(11.5)$ & \\
\hline BMI, median (Q1, Q3) & $44.0(40.4,48.8)$ & $46.7(42.5,51.9)$ & $51.0(45.1,60.5)$ & $<0.0001$ \\
\hline Female, n $(\%)$ & $1,227(76.3)$ & $3,087(81.9)$ & $341(67.7)$ & $<0.0001$ \\
\hline \multicolumn{5}{|l|}{ Patient race, $\mathrm{n}(\%)$} \\
\hline Caucasian (56 missing) & $1,426(89.7)$ & $3,271(87.6)$ & $464(92.2)$ & 0.0021 \\
\hline Hispanic (3 missing) & $81(5.0)$ & $229(6.1)$ & $18(3.6)$ & 0.0384 \\
\hline Current or recent smoker, $\mathrm{n}(\%){ }^{*}$ & $153(9.5)$ & $558(14.8)$ & $83(16.5)$ & $<0.0001$ \\
\hline
\end{tabular}


Table 2

Adverse Intraoperative Events by Surgical Approach

\begin{tabular}{|c|c|c|c|c|c|c|c|c|}
\hline \multirow[b]{2}{*}{ Characteristic } & \multicolumn{2}{|c|}{ Total $(\mathrm{n}=\mathbf{5 , 8 8 2})$} & \multicolumn{2}{|c|}{$\underline{\operatorname{LAGB}(n=1,608)}$} & \multicolumn{2}{|c|}{$\underline{\text { LRYGB }(n=3,770)}$} & \multicolumn{2}{|c|}{ ORYGB $(n=504)$} \\
\hline & $\mathbf{n}$ & $\%$ & $\mathbf{n}$ & $\%$ & $\mathbf{n}$ & $\%$ & $\mathbf{n}$ & $\%$ \\
\hline Adverse intraoperative event (any) & 294 & 5.0 & 48 & 3.0 & 209 & 5.5 & 37 & 7.3 \\
\hline \multicolumn{9}{|l|}{ Major adverse intraoperative events $*$} \\
\hline Anesthesia events & 51 & 0.9 & 17 & 1.1 & 32 & 0.8 & 2 & 0.4 \\
\hline Revision anastomosis ${ }^{\dagger}$ & 37 & 0.6 & 0 & 0.0 & 27 & 0.7 & 10 & 2.0 \\
\hline Instrument/equipment failure & 47 & 0.8 & 9 & 0.6 & 35 & 0.9 & 3 & 0.6 \\
\hline Liver injury & 24 & 0.4 & 7 & 0.4 & 16 & 0.4 & 1 & 0.2 \\
\hline Spleen injury & 13 & 0.2 & 2 & 0.1 & 8 & 0.2 & 3 & 0.6 \\
\hline Blood vessel injury & 4 & 0.1 & 1 & 0.1 & 2 & 0.1 & 1 & 0.2 \\
\hline Bowel injury & 52 & 0.9 & 1 & 0.1 & 46 & 1.2 & 5 & 1.0 \\
\hline
\end{tabular}

Only major events that are collected in both Longitudinal Assessment of Bariatric Surgery 1 and Longitudinal Assessment of Bariatric Surgery 2 are presented. For details, see Supplementary Tables 1 and 2, available online only.

${ }^{\dagger}$ Data for this item missing for 49 of 5,882 cases. 


\section{Table 3}

Relationship between 30-Day Major Adverse Complications and Incidence of an Adverse Intraoperative Event

\begin{tabular}{|c|c|c|c|c|c|c|c|}
\hline \multirow[b]{2}{*}{ Characteristic } & \multicolumn{2}{|c|}{ Total $(n=5,882)$} & \multicolumn{2}{|c|}{$\underline{\text { No AIE }(n=5,588)}$} & \multicolumn{2}{|c|}{ AIE $(n=294)$} & \multirow[b]{2}{*}{ p Value ${ }^{*}$} \\
\hline & $\mathbf{n}$ & $\%$ & $\mathbf{n}$ & $\%$ & $\mathbf{n}$ & $\%$ & \\
\hline Death & 16 & 0.3 & 15 & 0.3 & 1 & 0.3 & 0.56 \\
\hline DVT or PE & 21 & 0.4 & 20 & 0.4 & 1 & 0.3 & 0.96 \\
\hline Tracheal reintubation & 27 & 0.5 & 23 & 0.4 & 4 & 1.4 & 0.02 \\
\hline Endoscopy & 68 & 1.2 & 61 & 1.1 & 7 & 2.4 & 0.044 \\
\hline \multicolumn{8}{|l|}{ Operation } \\
\hline Tracheostomy & 12 & 0.2 & 10 & 0.2 & 2 & 0.7 & 0.06 \\
\hline Placement of percutaneous drain & 18 & 0.3 & 15 & 0.3 & 3 & 1.0 & 0.02 \\
\hline Abdominal reoperation & 147 & 2.5 & 133 & 2.4 & 14 & 4.8 & 0.01 \\
\hline Failure to be discharged by day 30 & 32 & 0.5 & 30 & 0.5 & 2 & 0.7 & 0.75 \\
\hline Composite end point ${ }^{\dagger}$ & 244 & 4.1 & 218 & 3.9 & 26 & 8.8 & $<0.0001$ \\
\hline From Fisher's exact test. & & & & & & & \\
\hline
\end{tabular}


Table 4

Multivariable Analysis of 30-Day Major Adverse Complications

\begin{tabular}{lcc}
\hline Characteristic & Relative risk (95\% CI) & p Value \\
\hline BMI (linear) & $*$ & 0.89 \\
\hline BMI (quadratic) & $*$ & 0.02 \\
\hline Inability to walk 200 feet (yes vs no) & $1.57(0.86-2.86)$ & 0.14 \\
\hline History of DVT (yes vs no) & $1.80(1.12-2.91)$ & 0.02 \\
\hline History of OSA (yes vs no) & $1.33(1.00-1.76)$ & 0.049 \\
\hline Procedure & & $<0.0001$ \\
\hline LRYGB vs LAGB & $3.60(2.23-5.80)$ & $<0.0001$ \\
\hline ORYGB vs LAGB & $3.54(1.86-6.74)$ & 0.0001 \\
\hline Surgeon volume (cases per year) & $0.95(0.90-1.00)$ & 0.07 \\
\hline Adverse intraoperative event (yes vs no) & $1.84(1.21-2.80)$ & 0.01 \\
\hline
\end{tabular}

Because BMI appears in the model as quadratic, the relative risk depends on the level of BMI and therefore a single relative risk could not be presented. BMI, body mass index; DVT, deep vein thrombosis; LAGB, laparoscopic adjusted gastric banding; LRYGB, laparoscopic Roux-en-Y gastric bypass; ORYGB, open Roux-en-Y gastric bypass; OSA, obstructive sleep apnea. 